\title{
El ajedrez en la enseñanza-aprendizaje de áreas científico-matemáticas, una revisión bibliográfica en el contexto español
}

\author{
Alicia Fernández-Oliveras \\ Universidad de Granada, España \\ alilia@ugr.es \\ Javier Ortega Dumont \\ Universidad de Granada, España \\ María Luisa Oliveras \\ Universidad de Granada, España
}

Recibido: 9 - febrero - 2018 / Aceptado: 31 - julio - 2018

\section{Resumen}

El juego es un proceso ancestral que permite desarrollar el pensamiento científico-matemático, lo que hace de los juegos unas herramientas didácticas de un indudable valor. Por ello, en los contextos educativos basados en enfoques etnocientíficos y etnomatemáticos, los juegos en general, y los juegos tradicionales en particular, deberían estar presentes. Esto es lo que pretendemos estudiar mediante la realización de una revisión bibliográfica centrada en el ajedrez, como ejemplo de juego tradicional. El presente trabajo resume dicha revisión bibliográfica que versa sobre las posibles aplicaciones educativas del ajedrez intentando analizar, además, las potencialidades de este tipo de recurso para mejorar las destrezas relacionadas con las ciencias experimentales y las matemáticas.

Palabras clave: Educación STEM (Science, Technology, Engineering and 
Mathematics), aprendizaje lúdico, aprendizaje basado en juegos, juegos tradicionales, etnomatemáticas.

\section{Abstract}

Play is an ancestral process that invites the development of scientific-mathematical thought, making games a didactic tool of unarguable value. Thus, in educational contexts based on ethnoscientific and ethnomathematical approaches, games in general, and traditional games in particular, should be encouraged. This is what we study though a literature review focusing on chess, as an example of a traditional game. The present work summarizes this review concerning the possible educational applications of chess seeking to analyse, furthermore, the potential of this type of resource to improve the skills related to experimental sciences and mathematics.

Keywords: Science, Technology, Engineering and Mathematics (STEM) Education, playful learning, game based learning, traditional games, ethnomathematics. 


\section{Introducción}

$\mathrm{E}$ 1 juego es un proceso que permite desarrollar el pensamiento científico-matemático, lo convierte a los juegos en herramientas didácticas de un indudable valor, como muestran algunas propuestas fundamentadas en la educación interdisciplinar e integral (Espinar, Fernández-Oliveras y Oliveras, 2014; Fernández-Oliveras, Molina y Oliveras, 2016). Bergen (2009) considera necesario fomentar el comportamiento lúdico y educar a través del juego para desarrollar las habilidades de las áreas STEM (Science, Technology, Engineering and Mathematics). La idea subyacente es que la investigación y el razonamiento de científicos y matemáticos se asemejan a la forma de pensar que tienen las personas cuando juegan de manera significativa. Según Bishop (1995), el juego es una de las prácticas matemáticas transculturales -además de contar, medir, diseñar, localizar, explicar- que pueden considerarse como prácticas propias de las matemáticas vivas o etnomatemáticas (Oliveras, 2005, 2006). Palhares (2012) considera a los juegos como uno de los artefactos a explorar para "descubrir pensamiento matemático oculto" (p. 88). Así, en los contextos educativos basados en enfoques interdisciplinares e integrales, los juegos en general, y los juegos tradicionales en particular, deberían de estar presentes. Y es por ello que, con una perspectiva etnomatemática e integradora, en este trabajo pretendemos analizar la literatura educativa en busca de relaciones entre el ajedrez y la educación científico-tecnológico-matemática. Esta revisión forma parte del Trabajo Final de Máster realizado por el segundo autor, bajo la dirección de las otras dos autoras.

\section{Metodología}

Se plantea la realización de una revisión bibliográfica sobre aquellas experiencias actuales llevadas a cabo en el aula y proyectos de innovación docente basados en el ajedrez, principalmente para la Educación Primaria en general y, para la educación científica y matemática, en particular, en el contexto español.

La dificultad reside en que este tipo de experiencias de aula y proyectos de innovación son estudios de gran interés pero que en la mayoría de ocasiones no se publican.

La realización del trabajo consta de varias fases: en primer lugar, de la identificación; en segundo lugar, de la selección; y finalmente, del análisis y la descripción de la información recogida sobre la temática.

\section{Resultados}

\section{Experiencias en el aula}

En la experiencia de Amigó (2009) se aplicó una metodología que consistió en realizar una breve introducción con un tablero mural para que los mismos estudiantes, apoyándose en el maestro como guía, descubrieran los distintos aspectos del 
ajedrez. Se llevaron a cabo partidas entre ellos, carreras de peones, la torre que come chocolate, etc. Se hicieron partidas amistosas entre las distintas clases del centro educativo, y algunas fichas de ejercicios sobre orientación espacial y razonamiento lógico o resolución de problemas mediante jaque y mate en una jugada. Además, se resolvieron preguntas de diversos tipos en un ordenador, entre ellas, algunas de cálculo mental utilizando el valor de las piezas. Finalmente, se hizo un juego a través del sistema de puntos con los cartones-pieza: se realizaba una ronda de preguntas y si se acertaba dicha pregunta, se obtenía un cartón con una pieza dibujada, la cual representa una cantidad de puntos (del 1-5), en función de su valor en el ajedrez. Posteriormente se procedía a jugar partidas con los compañeros y según el resultado y la actitud que se había mostrado durante la partida, se ganarían o perderían más o menos puntos.

García y Blanch (2016) diseńaron una propuesta de intervención educativa para favorecer la mejora de las competencias de lengua, tecnología y matemáticas, partiendo de una metodología enfocada en las TIC y en el juego del ajedrez. Esta metodología tuvo en cuenta, entre otras cosas, el contexto educativo, tareas que consiguieran lograr objetivos de aprendizaje, la planificación de las sesiones orientadas al aprendizaje constructivista y, por último, la configuración del método de evaluación.

Fernández, Pallarés y Gairín (2010) llevaron a cabo una serie de actividades para favorecer el proceso de enseñanza-aprendizaje mediante el uso del ajedrez, presentando juegos de dados, con tablero, con cartas de la baraja, el dominó, el hexágono, la diana y el diagrama (Figura 1).

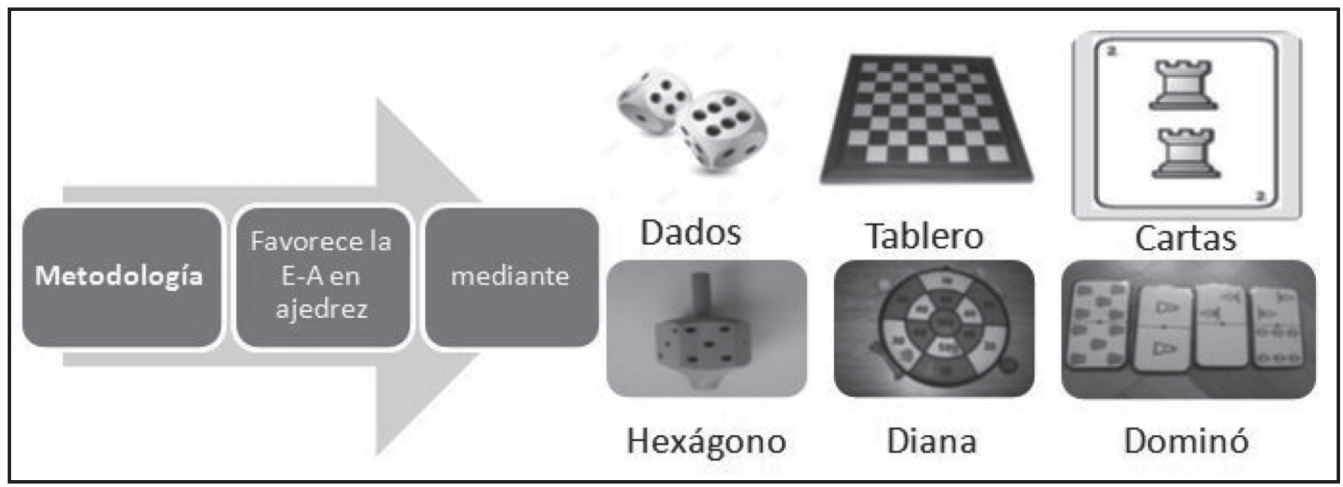

Figura 1. Metodología y materiales utilizados en juegos relacionados con el ajedrez en el trabajo de Fernández, Pallarés y Gairín (2010), donde "E-A" es "enseñanza-aprendizaje” (Elaboración propia).

Este material intenta aportar herramientas para proveer en la enseñanza una dimensión más motivadora y lúdica, proporcionando más recursos ajedrecísticos a la metodología de la enseñanza de la matemática para dar al currículo una forma más transversal, más dinámica y útil para la vida cotidiana (Fernández, Pallarés, y Rodrí- 
guez, 2004; Fernández, Rodríguez y Sánchez, 2004 citado en Fernández, Pallarés y Gairín, 2010).

Nortes y Nortes (2015) propusieron también actividades como contar el número de cuadrados que posee un tablero de ajedrez para encontrar la fórmula y posteriormente poder extrapolarla a otras dimensiones. Pueden trabajarse números mayores a través de la leyenda del ajedrez de los granos de trigo, por ejemplo, pidiendo a los alumnos que calculen las dimensiones que debería tener una caja donde poder guardar el trigo. Además, se pueden hacer juegos con poliminós, cuadrados mágicos o realizar recorridos con las diferentes fichas (rey, dama, alfil, caballo y torre) por el tablero, sin repetir ninguna casilla y de diferentes formas.

Maz-Machado y Jiménez-Fanjul (2012) realizaron algo parecido, proponiendo actividades que se pudieran integrar dentro de una metodología para trabajar patrones en matemáticas. Para ello, se utiliza un tablero de ajedrez y sus piezas. Ambos autores tienen en cuenta que, en primer lugar, los niños deben aprender a discernir entre filas, columnas y diagonales. En segundo lugar, se procede a enseñarles las distintas piezas que existen en ajedrez con sus respectivos nombres y cómo éstas se mueven por el tablero. Para la siguiente actividad necesitarán saber el movimiento que realiza el alfil, la dama y el caballo, ya que deben colocar estas piezas y colorear sus patrones de movimiento.

Callís, Falgás, Saurina, Serra y Amigó (2014) propusieron un proyecto bajo el lema "Observo, pienso y juego". Estas tres palabras establecen el proceso didáctico mediante el cual se pretende que el alumnado integre un hábito de trabajo reflexivo e interiorizado ante cualquier situación para resolver problemas de todo tipo.

Baubeg, Apostol, Flesner y Gliga (2013) desarrollaron un proyecto durante diez sesiones, donde se les explicó el movimiento de las piezas de ajedrez, jaques mate, tácticas y finales básicos. Cada sesión estaba compuesta por cuatro partes: una expositiva (película corta animada que ilustraba conceptos del ajedrez usando una metáfora), la segunda y tercera, interactivas y la última, de evaluación. Las partes interactivas podían ser de tres formas distintas: un tutorial (alta interactividad, pero baja creatividad), un ejemplo comentado (alta interactividad y creatividad algo mayor) o un problema de ajedrez (alta interactividad y creatividad). La evaluación consistía en un seguimiento del progreso en las partes anteriores.

\section{Proyectos de innovación docente}

El proyecto de Marín (2014) pretendía mejorar las siguientes destrezas: memoria, razonamiento lógico, resolución de problemas, concentración e imaginación, mediante el ajedrez como herramienta de aprendizaje transversal.

Villar (2011) fija como objetivos principales que los alumnos aprendan las reglas básicas del juego, las practiquen jugando y aprendan las estrategias para mejorar su capacidad de resolver problemas matemáticos. Además, pretende que su proyecto sirva para desconectar de la metodología tradicional. 
García y Blanch (2016), mediante una aplicación de ajedrez llamada Tecnochess, pretenden trabajar las competencias en lengua, tecnología y matemáticas, a través de un aprendizaje dinámico y lúdico, que pueda ser más motivador, utilizando el talento innato del alumnado con los juegos, y apostando un ambiente dinámico, motivador y tranquilo para estimular el aprendizaje.

Torres (2015) realizó un proyecto introduciendo el ajedrez con la intención de que los estudiantes perfeccionaran sus destrezas de cálculo numérico, razonamiento, pensamiento lógico, reflexivo y crítico, a la hora de proceder a resolver un problema matemático (Figura 2).

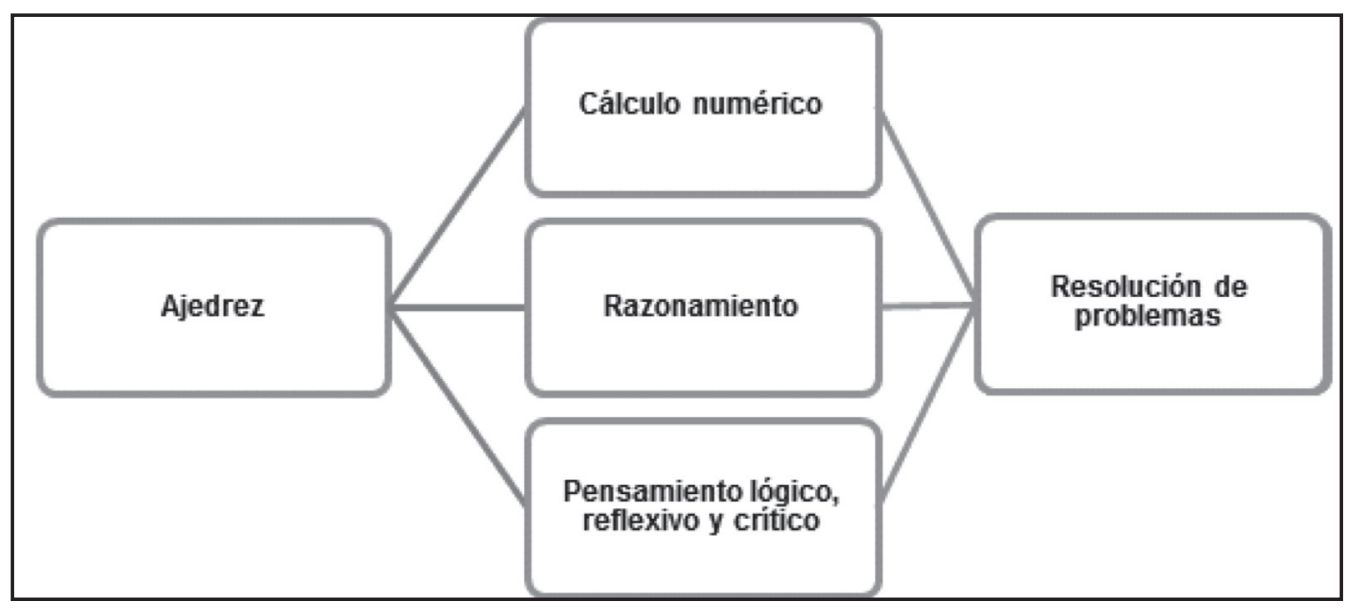

Figura 2. Destrezas que permite desarrollar el ajedrez como recurso para la resolución de problemas matemático-científicos según Torres (2015) (Elaboración propia).

El proyecto de innovación del I.E.S. José Ma Pereda (Troyano, 2016) pretendió desarrollar las capacidades intelectuales de sus estudiantes instaurando unos hábitos de esfuerzo, superación y mejora, consolidando la iniciativa personal, el sentido crítico y la autoestima, y proporcionando prácticas lúdicas compartidas entre iguales y con otros grupos de distintas edades.

Un proyecto de innovación de la Consejería de Educación y Ciencia de la Junta de Andalucía (2010), titulado "Ajedrez para todos: aprender jugando, jugar aprendiendo" propone objetivos a alcanzar para las diferentes partes que participan dentro de la comunidad educativa (Figura 3): alumnado, profesorado, centro educativo y familias, lo cual no se suele tener en cuenta. Durante la realización del mismo, a los alumnos se les pretende animar en la participación activa de su propio aprendizaje y atraerles de manera motivadora y lúdica hacia los contenidos de las distintas áreas curriculares a través del ajedrez. La tarea del centro a grandes rasgos será la de organizar y suministrar al equipo docente de los materiales y herramientas que se requieran para su implementación; insertar tanto en el proyecto de centro como en el cu- 
rricular, las diferentes propuestas pedagógicas que se pongan en marcha; trabajar de forma activa con las instituciones educativas y divulgar el trabajo que se ha realizado dentro de la comunidad escolar y con otros centros educativos. Finalmente, a las familias se les quiere comunicar los avances del proyecto; inducir a la participación en las actividades organizadas e integrarlas en el proceso educativo, permitiéndoles colaborar con sus hijos en las actividades llevadas a cabo en el aula.

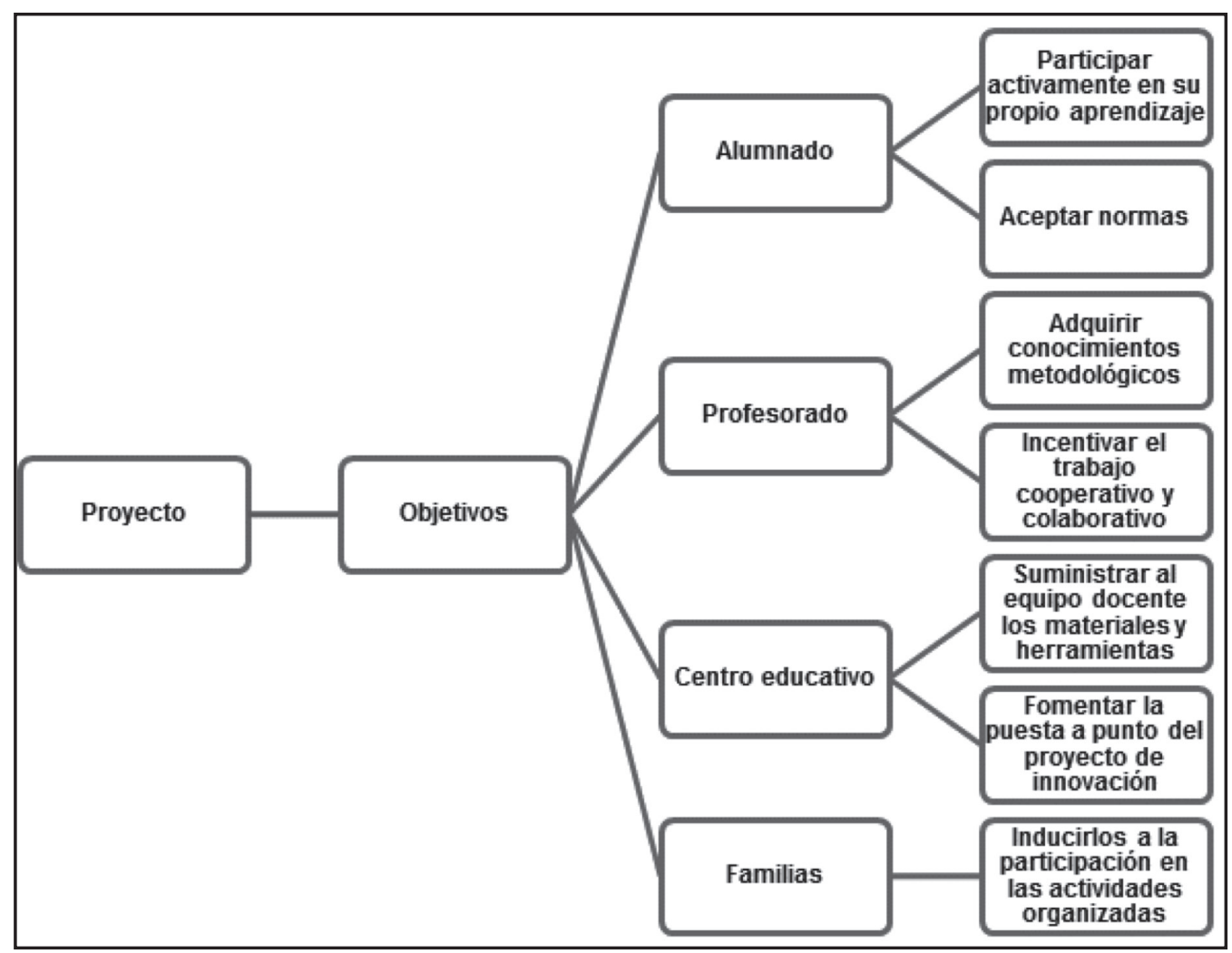

Figura 3. Objetivos que se pretenden conseguir con el proyecto de la Junta de Andalucía "Ajedrez para todos: aprender jugando, jugar aprendiendo" (Consejería de Educación, 2010) en cada uno de los agentes que intervienen en el proceso educativo (Elaboración propia).

El proyecto de Saucedo y Aldaba (2016) se propone desarrollar la convivencia sana a través del ajedrez, enseńando reglas para comportarse de acuerdo a las normas sociales.

Por último, el proyecto de innovación de Fernández y Pallarés (2009) buscaba concienciar a las familias y atraer a los niños a que descubran y conozcan el ajedrez.

\section{Conclusiones}

Mediante el trabajo realizado se ha logrado identificar fuentes de información sobre el ajedrez como herramienta de enseñanza-aprendizaje y su uso en el aprendizaje lúdico de las ciencias experimentales y las matemáticas. 
Según los resultados se puede observar cómo el ajedrez es una muy buena herramienta para las áreas de las ciencias experimentales y las matemáticas (STEM) así como para otras que aparentemente no guardan mucha relación, como el lenguaje y las ciencias sociales, pero que en un enfoque de enseñanza integral deben ir de la mano de las primeras.

\section{Referencias}

Amigó, M. (2009). Dame la mano. El ajedrez como herramienta educativa. Educared. https://rondalles.files.wordpress.com/2012/04/elajedrezcomoherramientaeducativa.pdf

Bergen, D. (2009). Play as the learning medium for future scientists, mathematiciams, and engineers. American journal of play, 1, 413-428.

Baubeg, B., Apóstol, S., Flesner, P. I., y Gliga, F. (2013). Chess in school- A blended learning pilot project. In Conference proceedings of eLearning and Software for Education (eLSE) (No. 01, pp. 220-225). Universitatea Nationala de Aparare Carol I. file://C:/Users/USUARIO/Downloads/CEEOL\%20Article.pdf

Bishop, A. (1995). Educando a los «culturizadores matemáticos». Uno: Revista de Didáctica de las Matemáticas, 6, 7-12.

Callís, J., Falgás, M., Saurina, C., Serra, J. y Amigó, M. Ajedrez. (2014). Ajedrez. Un recurso educativo para ayudar a mejorar aprendizajes y conductas. Aula de Innovación Educativa. 235, 43-48.

Consejería de Educación y Ciencia de la Junta de Andalucía (2010). Ajedrez para todos: aprender jugando, jugar aprendiendo. El ajedrez como herramienta pedagógica en el aula.

Espinar, G., Fernández-Oliveras, A., y Oliveras, M. L. (2014). El Ouril como ejemplo del uso de los juegos culturales para la enseñanza globalizadora de las matemáticas. Revista Electrónica de Investigación y Docencia Creativa ReiDoCrea, 3(29), 2445-256.

Fernández, J., Pallarés, M. R., y Gairín, J. (2010). Material didáctico, con recursos de ajedrez, para la enseñanza de las matemáticas. Uno: Revista de Didáctica de las Matemáticas, 17(54), 103-119.

Fernández, J., Pallarés, M. R., y Rodríguez, J. R. (2004). Ajedrez transversal. Aula de Innovación Educativa, 13(130), 65-68. http://www.paretana.com/zip/Ajedrez\%20transversal\%20_Sin\%20fotos_.pdf

Fernández, J., y Pallarés, M. R. (2009). Cómo sensibilizar la escuela hacia el ajedrez. Gestión de Centros. http://dim.pangea.org/revistaDIM15/docs/sensibilizarlaescuelahaciaelajedrez.pdf

Fernández-Oliveras, A., Molina, V. y Oliveras, M.L. (2016). Estudio de una propuesta lúdica para la educación científica y matemática globalizada en infantil. Revista Eureka sobre Enseñanza y Divulgación de las Ciencias, volumen 13(2), 373-383. 
García, H. y Blanch, A. (2016). Tecnochess. Una propuesta didáctica para trabajar las competencias lingüística, tecnológica y matemática mediante el juego del ajedrez y las TIC. RIITE. Revista Interuniversitaria de Investigación en Tecnología Educativa, 1, 39-51.

Marín, R. (2014). Proyecto Innovación Ajedrez en la Malla Curricular. Universidad de Chile. http://www.lms.cl/images/PDF/ProyPedagogicos/proyectoajedrezenelaula.pdf

Maz-Machado, A. y Jiménez-Fanjul, N. (2012). Ajedrez para trabajar patrones en matemáticas en Educación Primaria. Épsilon-Revista de Educación Matemática, 29 (81), 105-111.

Nortes, R., y Nortes, A. (2015). El ajedrez como recurso didáctico en la enseñanza-aprendizaje de las matemáticas. Números, Revista de Didáctica de las Matemáticas, 89, 9-31.

Oliveras, M. L. (2005). Microproyectos para la educación intercultural en Europa. Uno: Revista de Didáctica de las Matemáticas, 11(38), 70-81.

Oliveras, M. L. (2006). Etnomatemáticas: de la multiculturalidad al mestizaje. En J. Goñi, M. Albertí, S. Burgos, R. Díaz, G. Dominguez, G. Fioriti, et al. (Eds.), Matemática e Interculturalidad (pp. 117-149). Barcelona, España: Graó.

Palhares, P. (2012). Mathematics education and ethnomathematics. A connection in need of reinforcement. Journal of Research in Mathematics Education, 1(1), 79-92.

Saucedo, M. D., y Aldaba, J.R. (2016). El ajedrez como estrategia vital didáctica para favorecer la sana convivencia en educación preescolar. En A. Barraza, T. J. Cárdenas (Coords.), Proyectos de innovación didáctica, en, y desde, los diferentes niveles educativos (pp. 8-22). México: Instituto Universitario Anglo Español.

Torres, I. (2015). El ajedrez como estrategia didáctica vital para la enseñanza de las matemáticas en la escuela primaria. Instituto Universitario Anglo Español. Proyectos de innovación didáctica para la mejora de la práctica docente, 126.

Troyano, A. (2016). Proyecto de innovación pedagógica de ajedrez educativo. Esos viejos juegos clásicos. http://iespereda.es/proyecto_ajedrez educativo.html

Villar, R. (2011). Matemáticas y ajedrez. (Trabajo fin de Máster). Universidad de la Rioja. España. http://biblioteca.unirioja.es/tfe_e/TFE000134.pdf 